\title{
Biologia reprodutiva de Macrobrachium jelskii (Crustacea, Decapoda, Palaemonidae) no Rio São Francisco, Minas Gerais, Brasil
}

\author{
Marcelo R. da S. Soares, Lídia M. Y. Oshiro \& Júlio C. Toledo
}

Estação de Biologia Marinha, Universidade Federal Rural do Rio de Janeiro, Rua Sereder s/nº Itacuruçá, Mangaratiba, 23880-000, Rio de Janeiro, Brasil. (marcelorssoares@gmail.com)

\begin{abstract}
Reproductive biology of the shrimp Macrobrachium jelskii (Crustacea, Decapoda, Palaemonidae) in São Francisco River, Minas Gerais, Brazil. The objective was evaluate the reproductive biology of Macrobrachium jelskii (Miers, 1877) in São Francisco River, with emphasis on sex ratio, reproductive period, sexual maturity and fertility. The samples were collected monthly from March 2005 to February 2006 except April and October 2005, in two sites in São Fransico River, upstream of the Três Marias Dam (Pirapitinga Ecological Station) and downstream of the dam, located in Três Marias, central region of Minas Gerais state, Brazil. The netting mouth had $1 \mathrm{~m}^{2}$ and the mesh $1.42 \mathrm{~mm}$ that it was passed under the marginal vegetation and the water next to the bank during 30 minutes. In the laboratory, specimens were identified, measured and sexed. A total of 2,945 specimens were captured in the upstream, 1,064 males and 1,857 females (1,653 non-ovigerous and 204 ovigerous) and 1,973 individuals in the downstream, 1,120 males and 841 females (643 non-ovigerous and 198 ovigerous). The sex ratio differed significantly for females of $M$. jelskii in upstream $\left(0.57, \chi^{2}=215.28, p<0.01\right)$, and males in the downstream $(1.33, \chi 2=39.69, p<0.01)$. In both sites the reproductive period was a continuous - seasonal with the peak occurring in January and November for shrimps of upstream and downstream, respectively. The mean fecundity of $M$. jelskii was $37 \pm 14$ eggs (5-78 eggs) in the upstream of the dam and 35 \pm 14 eggs (5-69 eggs) in the downstream of the dam. The sex ratio showed deviation to males of $M$. jelskii in the downstream suggesting that females presenting behavior of hiding in the marginal vegetation. And in the upstream the sex was deviate to females, demonstrating the actual proportion of males and females of the population, probably because females do not find places to hide. The eggs size carried by females downstream was influenced by the low water temperatures from the dam.
\end{abstract}

KEYWORDS. Freshwater shrimp, sex ratio, reproductive period, sexual maturity and fertility.

RESUMO. O objetivo foi avaliar a biologia reprodutiva do camarão- sossego Macrobrachium jelskii (Miers, 1877) no Rio São Francisco, com ênfase na razão sexual, período reprodutivo, maturidade sexual e fecundidade. As coletas foram realizadas mensalmente entre março de 2005 e fevereiro de 2006 exceto nos meses de abril e outubro, nos dois pontos de coleta do Rio São Francisco, Represa de Três Marias (Estação Ecológica de Pirapitinga), a montante da barragem da usina hidroelétrica de Três Marias e a jusante da barragem, localizados no município de Três Marias, região central de Minas Gerais, Brasil. O puçá de $1 \mathrm{~m}^{2}$ de boca e malha de 1,42 $\mathrm{mm}$ foi passado sob a vegetação marginal e na água à margem dos dois pontos de coleta, por um coletor em um período de até 30 minutos. Em laboratório, os espécimes foram identificados, mensurados e sexados. Um total de 2.945 espécimes foi analisado a montante, no qual correspondem a 1.064 machos e 1.857 fêmeas (1.653 não ovígeras e 204 ovígeras) e 1.973 a jusante, onde 1.120 machos e 841 fêmeas (643 não ovígeras e 198 ovígeras). A razão sexual diferiu significativamente a favor de fêmeas de $M$. jelskii a montante $\left(0,57 ; \chi^{2}=215,28 ; p<0.01\right)$ e a favor dos machos a jusante $(1,33 ; \chi 2=39,69 ; p<0.01)$. Ambas as populações possuem um período reprodutivo contínuo-sazonal com pico em janeiro e novembro para os camarões a montante e a jusante, respectivamente. A fecundidade média de $M$. jelskii foi de $37 \pm 14$ ovos ( 5 a 78 ovos) a montante da barragem e de $35 \pm 14$ ovos ( 5 a 69 ovos) a jusante. A razão sexual desviada para os machos de M. jelskii à jusante sugere que as fêmeas apresentaram o comportamento de se abrigarem entre as vegetações marginais. A razão sexual desviada para as fêmeas a montante demonstra a real proporção de machos e fêmeas, que pode ser considerada para a espécie no Rio São Francisco. O tamanho dos ovos carregados pelas fêmeas a jusante foi influenciado pelas baixas temperaturas da água proveniente da represa.

PALAVRAS-CHAVE. Camarão de água doce, razão sexual, período reprodutivo, maturidade sexual, fertilidade.

Macrobrachium jelskii (Miers, 1877) é um crustáceo de pequeno porte, conhecido popularmente no Brasil como camarão-sossego (Bastos \& PaIva, 1959). Distribui-se desde Trinidad, Venezuela, Guiana, Suriname, Guiana Francesa, Bolívia até o Brasil (Amapá, Pará, Amazonas, Maranhão, Ceará, Rio Grande do Norte, Paraíba, Pernambuco, Alagoas, Sergipe, Bahia, Minas Gerais, Espírito Santo, Mato Grosso, São Paulo e Santa Catarina) (Melo, 2003) e Argentina (Collins, 2000).

Ao contrário da maioria das espécies de Macrobrachium Bate, 1868, M. jelskii independe fisiologicamente de água salobra para completar seu ciclo vital, ficando restrita ao ambiente de água doce e, de acordo com Montoya (2003), a espécie aparece associada a raízes de plantas aquáticas, que fornecem recursos nutricionais e proteção para as fêmeas ovígeras e para o desenvolvimento dos estágios larvais da espécie. Segundo Melo (2003), alimentam-se de larvas de insetos, diatomáceas e outras algas, além de grãos do sedimento. MaGALHÃEs (2000) afirmou que os indivíduos apresentam desenvolvimento larval abreviado e as fêmeas carregam um número reduzido de ovos volumosos.

São poucas as contribuições sobre a biologia e ecologia de M. jelskii. Barros-Alves et al. (2012) no Rio Grande, noroeste do estado de Minas Gerais, Mossolin et al. (2013) na região central de São Paulo e PAIVA \& BARRETO (1960) no Ceará contribuíram com informações sobre a biologia, com ênfase nos aspectos reprodutivos, e Bastos \& Paiva (1959), também no Ceará, estudaram sobre o consumo de oxigênio do camarão-sossego. No 
Amazonas, MagalHães (2000) verificou o desenvolvimento larval abreviado e, Gamba (1997) na Venezuela, obteve informações sobre a biologia reprodutiva da espécie.

No estado de Minas Gerais, esta espécie é considerada rara, entretanto tem aparecido com frequência e abundância no Rio São Francisco, onde se localiza a Represa de Três Marias, não sendo conhecidas pesquisas realizadas com esta espécie na região central de Minas Gerais. Este trabalho teve como objetivo obter informações sobre a biologia reprodutiva de $M$. jelskii em uma região com interferência de barragem.

\section{MATERIAL E MÉTODOS}

O estudo foi realizado no Rio São Francisco, localizado no município de Três Marias, região central de Minas Gerais, durante o período de março de 2005 a fevereiro de 2006 (Fig. 1).

As coletas foram realizadas em dois pontos distintos do Rio São Francisco, um na Represa de Três Marias (Estação Ecológica de Pirapitinga), a montante da barragem da usina hidroelétrica de Três Marias (Três Marias, $\left.18^{\circ} 21^{\prime} 49^{\prime \prime} \mathrm{S}, 45^{\circ} 19^{\prime} 55^{\prime \prime} \mathrm{O}, 570 \mathrm{~m}\right)$ e outro a jusante da barragem (Três Marias, $18^{\circ} 12^{\prime} 30^{\prime}$ 'S , 45 $5^{\circ} 15^{\prime} 50^{\prime} \mathrm{O}, 517 \mathrm{~m}$ ), distando aproximadamente $18,64 \mathrm{~km}$ um do outro, com o auxílio da CEMIG GT/CODEVASF (Companhia Energética de Minas Gerais/Companhia de Desenvolvimento do Vale do São Francisco) e do acordo de cooperação da UFRRJ (Universidade Federal Rural do Rio de Janeiro) com o IBAMA/ESEC de Pirapitinga/MG (Instituto Brasileiro do Meio Ambiente e dos Reursos Naturais Renováveis/ Estação Ecológica de Pirapitinga/Minas Gerais) (Fig. 1).

A montante as coletas foram efetuadas na margem da Estação Ecológica de Pirapitinga, onde o ambiente é lêntico. Esse ponto caracteriza-se por possuir margem com pouca vegetação, formada basicamente por gramíneas, principalmente na estação seca-fria, quando o nível de água da Represa está mais baixo.

As coletas a jusante da barragem da Usina hidroelétrica de Três Marias foram realizadas num ponto distante, aproximadamente 500 metros da barragem, onde o fluxo de água é intenso devido à saída da água da represa. Este ponto de coleta caracteriza-se por possuir uma vasta vegetação marginal com grande quantidade de plantas aquáticas.

Os pontos de coleta diferem em relação ao tipo de ambiente, um é lêntico e o outro é lótico e, em relação à vegetação marginal, no ponto a montante a vegetação é bem reduzida, com poucos refúgios para os camarões e a jusante a vegetação é vasta e com muitos refúgios para a espécie.

O nível da água tanto a montante quanto a jusante variou de acordo com a época do ano, sendo esta variação mais visível a montante, no reservatório da Represa.

As coletas foram realizadas mensalmente entre março de 2005 a fevereiro de 2006, exceto nos meses de abril e outubro. Utilizou-se um puçá de $1 \mathrm{~m}^{2}$ de boca

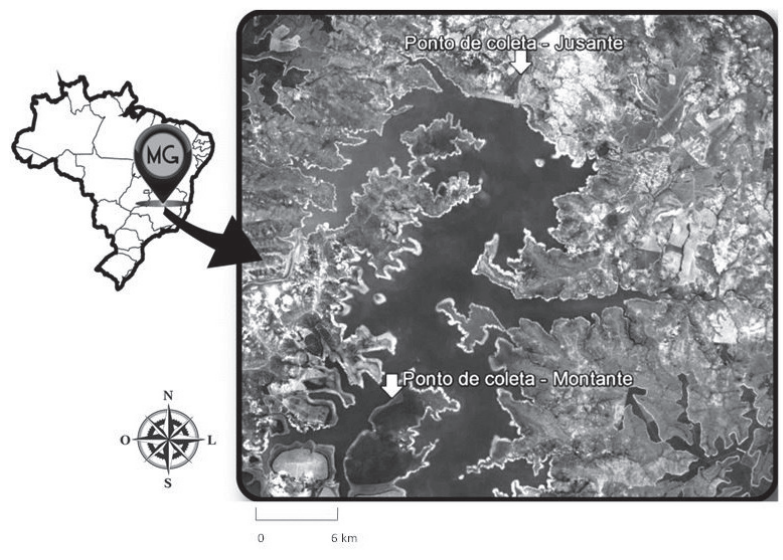

Fig. 1. Localização dos pontos de coleta no Rio São Francisco, a montante da barragem da Represa de Três Marias (Estação Ecológica de Pirapitinga) $\left(18^{\circ} 21^{\prime} 49^{\prime} \mathrm{S} 45^{\circ} 19^{\prime} 55^{\prime} \mathrm{O}\right)$ e a jusante (18॰12'30”S $\left.45^{\circ} 15^{\prime} 50^{\prime} \mathrm{O}\right)$, município de Três Marias, região central do estado de Minas Gerais, Brasil.

e malha de $1,42 \mathrm{~mm}$, que foi passado sob a vegetação marginal e na água próximo à margem nos dois pontos de coleta por um coletor em um período de até 30 minutos. A temperatura da água foi registrada mensalmente com auxílio de um termômetro digital.

Os exemplares capturados em ambos os locais foram acondicionados em recipientes com formaldeído a $10 \% \mathrm{e}$ etiquetados no laboratório da Estação de Hidrobiologia e Piscicultura de Três Marias (CODEVASF), sendo posteriormente levados ao laboratório da Estação de Biologia Marinha da UFRRJ (EBM/UFRRJ), em Itacuruçál $\mathrm{RJ}$, onde foram processados e depois depositados na coleção de crustáceos do Museu Nacional/Universidade Federal do Rio de Janeiro (números de tombo dos materiais depositados: MNRJ25235; MNRJ25236; MNRJ25237).

No laboratório da EBM/UFRRJ, após a identificação e determinação do sexo de cada indivíduo, foram tomados as medidas do comprimento total (CT, medida entre as extremidades distais do rostro e do telso) e comprimento do cefalotórax $(\mathrm{CC}$, distância entre a órbita ocular ao bordo posterior do cefalotórax), com um paquímetro digital $(0,01$ $\mathrm{mm}$ ). Foram considerados indivíduos jovens, aqueles que apresentaram o comprimento total inferior ao do menor macho com presença do apêndice masculino. As fêmeas ovígeras foram identificadas pela presença de ovos aderidos aos pleópodos.

Foram definidas duas estações sazonais: estação quente e úmida (chuvosa-quente) - que tem como característica um período com alto índice de chuva e com altas temperaturas, que ocorre entre os meses de outubro a março; estação fria e seca (seca-fria) - caracterizada por baixo índice de chuva e temperaturas mais baixas, que ocorre entre os meses de abril a setembro.

O período reprodutivo foi determinado através da frequência relativa das fêmeas ovígeras capturadas ao longo dos meses de coleta, de acordo com PinHeIro \& Fransozo (2002), que sugeriram uma reclassificação para o período reprodutivo proposto inicialmente por SASTRY (1983) para 
crustáceos. Nesta reclassificação, a reprodução é contínua quando fềmeas ovígeras ou fêmeas com gônadas maduras estão presentes durante $\mathrm{o}$ ano todo, com frequências mensais semelhantes; se as fêmeas ovígeras estão presentes durante todo o ano com períodos de atividade reprodutiva mais alta, então a reprodução é contínua-sazonal; e se a presença de fêmeas ovígeras ocorre em um período determinado, a reprodução é sazonal.

A maturidade sexual individual foi determinada pelo comprimento total da menor fêmea ovígera capturada, enquanto a maturidade sexual populacional foi estimada para a classe de comprimento total em que se encontravam $50 \%$ do número de fêmeas ovígeras da população (VAZzoler, 1981). As classes de comprimento foram calculadas segundo BeIGUELMAN (2002).

Para o estudo da fecundidade foram utilizadas 198 fêmeas ovígeras do ponto a montante e 192 do ponto a jusante, que incubavam ovos em diferentes estágios de desenvolvimento embrionário. Estas foram separadas e acondicionadas individualmente, e posteriormente os ovos foram retirados da câmara incubadora com auxílio de pinças e contados com um contador manual. A relação entre a quantidade máxima de ovos (fecundidade máxima) carregados pelas fêmeas de diversos tamanhos (variável morfométrica) foi analisada através de uma regressão linear simples (FUROTA, 1996) com nível de significância de 5\% (ZAR, 2010), adotando-se como variável dependente o número de ovos e como variável independente, o comprimento total (mm).

Para determinar o tamanho dos ovos, de cada fêmea ovígera foram mensurados aleatoriamente vinte ovos. As medidas (diâmetro maior e menor) foram efetuadas sob uma ocular micrométrica. Os ovos foram analisados quanto ao estágio embrionário, sendo consideradas duas fases embrionárias distintas: fase inicial (ovos sem pigmentação dos olhos compostos) e fase final (ovos com pigmentação dos olhos compostos).

Para verificar diferenças em relação à temperatura da água a montante e a jusante no Rio São Francisco, foi utilizado o Teste t de Student com 5\% de significância (ZAR, 2010).

A razão sexual foi determinada através das proporções mensal e total, sendo utilizado o teste do Qui-quadrado, com 5\% de significância, para verificar diferenças entre estas proporções (ZAR, 2010). Para a comparação do tamanho dos ovos entre as duas fases de desenvolvimento embrionário dos dois locais de coleta foi utilizada a análise de variância (ANOVA) seguida do teste de Tukey (Q) com nível de significância de 5\% (ZAR, 2010). A normalidade dos grupos de dados utilizados no presente estudo foi confirmada através do teste de normalidade de Kolmogorov-Smirnov com 5\% de significância (ZAR, 2010).

\section{RESULTADOS}

A montante foi capturado um total de 2.945 indivíduos, dos quais 1.064 (36,13\%) machos, 1.857 (63\%) fêmeas e $24(0,81 \%)$ juvenis. Das 1.857 fêmeas capturadas, 204 (10,98\%) eram ovígeras. A jusante foram capturados 1.973 indivíduos, dos quais $1.120(56,76 \%)$ machos, 841 $(42,62 \%)$ fêmeas e $12(0,61 \%)$ juvenis. Das 841 fêmeas capturadas, 198 (23,54\%) eram ovígeras. Os indivíduos apresentaram comprimento total de 5,07 a $51,21 \mathrm{~mm}$ e 11,30 a 50,34 $\mathrm{mm}$ a montante e a jusante, respectivamente.

A razão sexual (M:F) para a população de M. jelskii a montante foi de $0,57\left(X^{2}=215,28 ; p<0,01\right)$ e a jusante de $1,33\left(X^{2}=39,69 ; p<0,01\right)$ (Tab. I). As fêmeas ovígeras foram capturadas durante todos os meses de coleta, com maior frequência em janeiro a montante e novembro a jusante, ambos no período chuvoso-quente (Figs 2, 3).

A maturidade sexual individual foi verificada aos $30,32 \mathrm{~mm}$ de comprimento total $(\mathrm{CC}=7,93 \mathrm{~mm})$ a montante e 35,24 $\mathrm{mm}(\mathrm{CC}=8,45 \mathrm{~mm})$ a jusante, enquanto a maturidade sexual populacional foi estimada para a classe de comprimento total de 39,23 a $41,73 \mathrm{~mm}$ no ponto a montante e a jusante do Rio São Francisco (Fig. 4).

A fecundidade média de foi de $37 \pm 14$ ovos, variando de 5 a 78 ovos por fêmea $(\mathrm{CT}=30,32$ a 50,64 $\mathrm{mm})$ a montante e de $35 \pm 14$ ovos, de 5 a 69 ovos (CT $=$ 35,24 a $48,72 \mathrm{~mm}$ ) a jusante. Houve associação entre o tamanho das fêmeas e o número máximo de ovos carregados por elas a montante $(F=18,49 ; p<0,01)$ (Fig. 5) e a jusante $(\mathrm{F}=46,52 ; \mathrm{p}<0,01)$ (Fig. 6); quanto maior a fêmea, maior é a quantidade de ovos carregados por ela.

Tab. I. Frequência absoluta e razão sexual de machos e fêmeas (M:F) de Macrobrachium jelskii (Miers, 1877) em cada mês de coleta, durante o período de março/2005 a fevereiro/2006 à montante e à jusante, Rio São Francisco, Minas Gerais, Brasil (*, p < 0,05).

\begin{tabular}{|c|c|c|c|c|c|c|c|c|}
\hline \multirow{2}{*}{ Mês } & \multicolumn{4}{|c|}{ Montante } & \multicolumn{4}{|c|}{ Jusante } \\
\hline & Macho & Fêmea & Razão sexual & $\mathrm{p}$ & Macho & Fêmea & Razão sexual & $\mathrm{p}$ \\
\hline Mar/2005 & 119 & 228 & $0,52 *$ & $<0,01$ & 126 & 38 & $3,31 *$ & $<0,01$ \\
\hline Mai/2005 & 193 & 238 & $0,81 *$ & $<0,05$ & 82 & 88 & 0,93 & $>0,05$ \\
\hline Jun/2005 & 169 & 377 & $0,45^{*}$ & $<0,01$ & 151 & 57 & $2,65^{*}$ & $<0,01$ \\
\hline $\mathrm{Jul} / 2005$ & 67 & 133 & $0,50 *$ & $<0,01$ & 116 & 70 & $1,66^{*}$ & $<0,01$ \\
\hline $\mathrm{Ago} / 2005$ & 66 & 128 & $0,51 *$ & $<0,01$ & 145 & 85 & $1,71^{*}$ & $<0,01$ \\
\hline Set/2005 & 90 & 86 & 1,05 & $>0,05$ & 94 & 56 & $1,68 *$ & $<0,01$ \\
\hline Nov/2005 & 66 & 122 & $0,54 *$ & $<0,01$ & 132 & 97 & $1,36^{*}$ & $<0,05$ \\
\hline Dez/2005 & 110 & 246 & $0,45^{*}$ & $<0,01$ & 86 & 115 & $0,75^{*}$ & $<0,05$ \\
\hline Jan/2006 & 67 & 135 & $0,50 *$ & $<0,01$ & 104 & 146 & $0,71 *$ & $<0,01$ \\
\hline $\mathrm{Fev} / 2006$ & 117 & 164 & $0,71 *$ & $<0,01$ & 84 & 89 & 0,94 & $>0,05$ \\
\hline Total & 1.064 & 1.857 & $0,57^{*}$ & $<0,01$ & 1.120 & 841 & $1,33 *$ & $<0,01$ \\
\hline
\end{tabular}



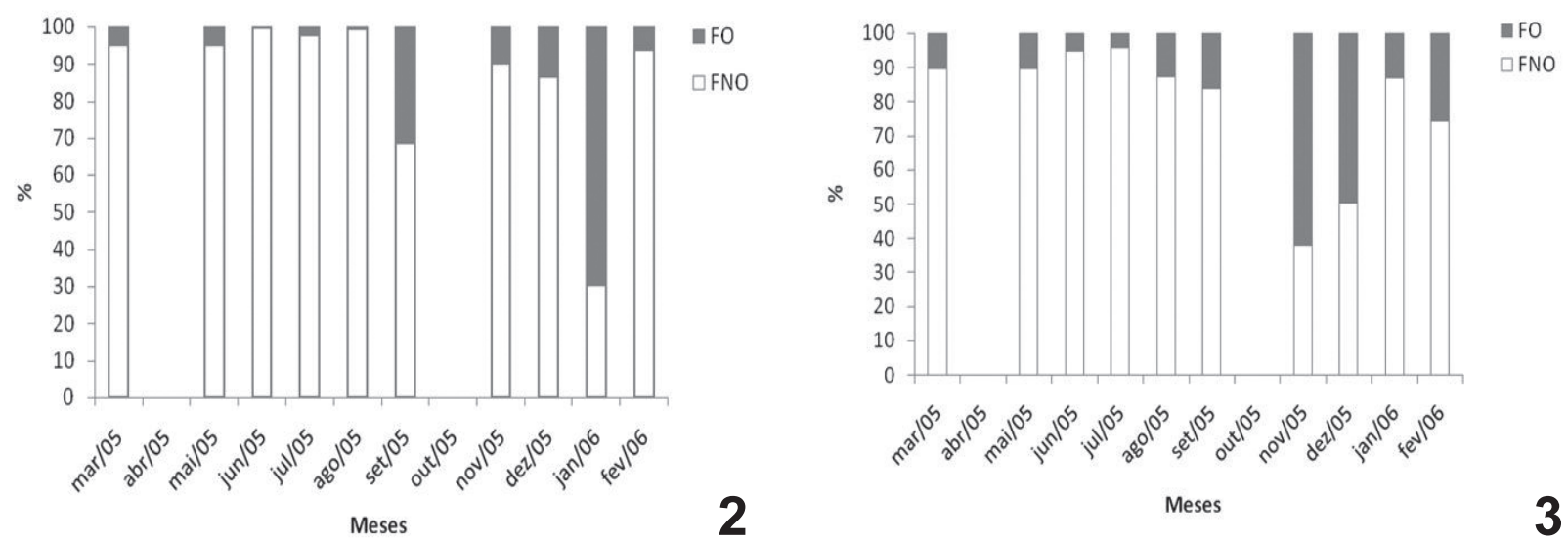

Figs 2, 3. Frequência relativa de fêmeas ovígeras (FO) e não ovígeras (FNO) de Macrobrachium jelskii (Miers, 1877) durante o período de março de 2005 a fevereiro de 2006, Rio São Francisco, Minas Gerais, Brasil: 2, a montante; 3, a jusante.

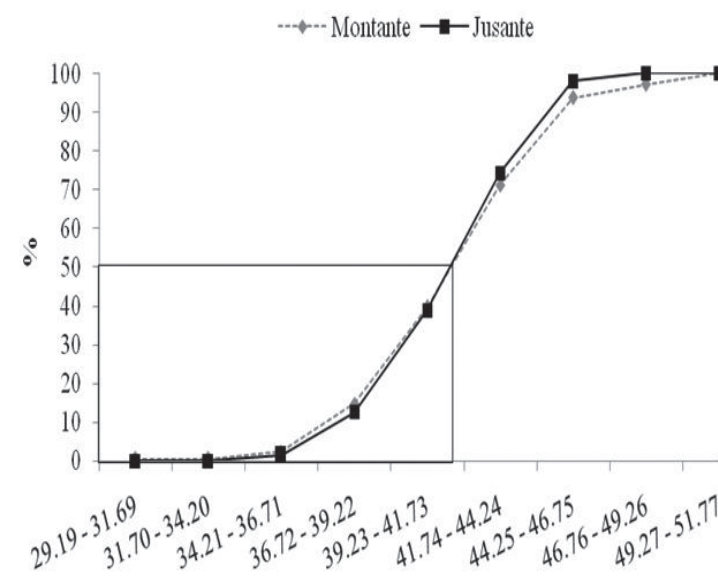

Fig. 4. Frequência relativa de fêmeas ovígeras de Macrobrachium jelskii (Miers, 1877) de acordo com as classes de comprimento total (mm) a montante e a jusante no Rio São Francisco, Minas Gerais, Brasil durante o período de março de 2005 a fevereiro de 2006.
Ao compararmos o tamanho dos ovos dos camarões, verificou-se que os camarões a jusante possuem ovos significativamente maiores (eixo menor $\mathrm{Q}=7,22 ; \mathrm{p}<0,01$ /eixo maior $\mathrm{Q}=4,68 ; \mathrm{p}<0,01) \mathrm{em}$ relação aos obtidos a montante. Em relação à forma, os ovos apresentaram formato elipsóide e suas dimensões demonstraram um aumento significativo entre as duas fases de desenvolvimento embrionária analisadas, a montante (eixo maior $\mathrm{Q}=15,60 ; \mathrm{p}<0,01 /$ eixo menor $\mathrm{Q}=11,04$; $\mathrm{p}$ $<0,01$ ) e a jusante (eixo maior $\mathrm{Q}=16,32 ; \mathrm{p}<0,01 /$ eixo menor $\mathrm{Q}=10,62 ; \mathrm{p}<0,01)$ (Tab. II).

\section{DISCUSSÃO}

A barragem de Três Marias, no rio São Francisco foi construída entre 1957 a 1960 e nessa época não se tinha registro de M. jelskii no local. Apenas no final da década de 1990 a espécie começou a ser observada e a hipótese mais

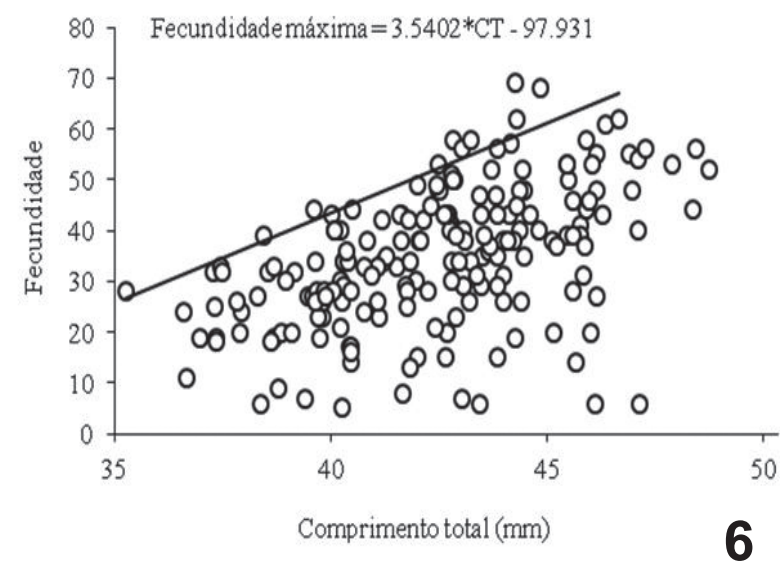

Figs 5, 6. Distribuição do número máximo de ovos (Fecundidade) de acordo com o comprimento total (mm) de Macrobrachium jelskii (Miers, 1877) do Rio São Francisco, Minas Gerais, Brasil, durante o período de março de 2005 a fevereiro de 2006: 5, a montante; 6, a jusante. 
Tab. II. Dimensões médias dos ovos ( $\mathrm{mm}$ ) na fase inicial e final de desenvolvimento embrionário de Macrobrachium jelskii (Miers, 1877) a montante e a jusante do Rio São Francisco, Minas Gerais, Brasil, durante o período de março/2005 e fe vereiro/2006 (*, p < 0,05).

\begin{tabular}{lccc}
\hline & & \multicolumn{2}{c}{ Dimensões médias dos ovos $(\mathrm{mm})$} \\
\cline { 2 - 4 } & \multicolumn{2}{c}{ Fase inicial } & Fase final \\
\cline { 2 - 4 } Jusante & Eixo menor & Eixo maior & Eixo menor \\
Montante & $1,59 \pm 0,06^{*}$ & $2,17 \pm 0,11^{*}$ & $1,68 \pm 0,11^{*}$ \\
\hline
\end{tabular}

aceita para o seu aparecimento é que os animais chegaram provenientes de criadouros que existiam às margens do rio São Francisco (Y. Sato, comum. pess.).

Os dados da Tab. I mostram que ambas populações não têm relação com a proporção sexual teórica esperada de $1: 1$, pois na população a montante a proporção foi desviada para as fêmeas e a jusante foi para os machos.

Gamba (1997) observou uma razão sexual populacional para M. jelskii de 0,58 na laguna de Pueblo de Mantecal, Venezuela e BArros-Alves et al. (2012) também registraram maior número de fềmeas na população do Rio Grande, Minas Gerais. Essas razões sexuais foram muito semelhantes à observada no ponto a montante do Rio São Francisco. Porém Mossolin et al. (2013) não verificou diferença significativa nas quantidades de machos e fềmeas em São Paulo. O maior número de fêmeas pode favorecer a um maior índice de fecundação, devido principalmente à característica de reprodução contínua da espécie.

WeNNER (1972) mencionou que razões sexuais desviadas da proporção esperada de 1:1 são amplamente difundidas entre os crustáceos. Segundo Barros-Alves et al. (2012), a razão sexual desviada para as fêmeas é comum em muitas espécies de Macrobrachium, como M. amazonicum (Heller, 1862), M. iheringi (Ortamann, 1897) e M. brasiliense (Heller, 1862), estudados respectivamente por Montoya (2003), Fransozo et al. (2004) e Mantelatto \& BARBosa (2005). Wilson \& Pianka (1963) afirmaram que o maior número de indivíduos para um dos sexos pode ser consequência das diferenças no tamanho, taxas de natalidade e mortalidade diferencial entre machos e fềmeas. E, de acordo com Botelho et al. (2001), outros fatores como as taxas de muda, dispersão, reprodução e migração diferencial também podem influenciar nessas diferenças.

No presente estudo, a obtenção de maior proporção de fêmeas durante praticamente todo o período de coleta a montante provavelmente está associado às características do local de coleta, com poucos refúgios para as fêmeas, possibilitando a obtenção de uma amostra que retrata melhor a distribuição entre os sexos desta população no rio São Francisco. A jusante, a maior captura de machos se deve provavelmente aos numerosos locais que possivelmente servem de abrigo para as fêmeas, na tentativa de se protegerem. Isso pode ocorrer devido à maior intensidade de predação sobre fêmeas maduras e ovígeras, pois segundo HOWARD \& LOWE (1984) essa intensa predação pode ser explicada não só pelo fato das fêmeas maduras atingirem tamanhos maiores que os machos, bem como por fatores que interferem nos mecanismos de escape de fêmeas ovígeras. E um desses fatores é a massa de ovos, que as tornam mais pesadas, e o outro, o volume dos ovos, que prejudica a rápida flexão do abdome. Esses mesmos autores afirmaram que na Austrália uma espécie de ave da ordem dos Ciconniformes, o coelheiro real (Patalea regia Gould, 1838), é o maior predador de M. intermedium (Stimpson, 1860), sendo a maior intensidade de predação incidente sobre fêmeas maduras e ovígeras dessa espécie.

Não somente as aves são consideradas predadoras de fêmeas maduras e ovígeras de palaemonídeos, como também alguns peixes, pois SouzA et al. (1996) constataram durante exames de conteúdos estomacais em peixes no arroio Sapucaia, que Hoplias malabaricus (Bloch, 1794), a traíra, como um predador potencial de Macrobrachium potiuna (Müller, 1880).

Os camarões a montante e a jusante apresentaram período reprodutivo contínuo-sazonal (Figs 2, 3), corroborando com Mossolin et al. (2013), que encontraram o período reprodutivo contínuo-sazonal, com maior atividade reprodutiva entre novembro e janeiro, meses com maior precipitação e temperatura (período chuvoso-quente). BARROS-ALVES et al. (2012) verificaram período reprodutivo contínuo para a espécie em Minas Gerais. PAIVA \& BARRETO (1960) registraram um período reprodutivo sazonal, entre abril e outubro, sendo mais intenso neste último, independente da estação chuvosa (janeiro-junho). GAMBA (1997) também encontrou um período reprodutivo sazonal, de maio a outubro, com altos índices de precipitação e temperatura da água. Esses autores verificaram um período reprodutivo para a espécie diferente do encontrado no presente estudo. As diferenças em relação aos períodos reprodutivos em Minas Gerais e São Paulo (variando de contínuo-sazonal a contínuo) e em relação ao Ceará e Venezuela (sazonal) podem ser inferidos às diferenças latitudinais.

Outros representantes de Macrobrachium como $M$. birai Lobão, Melo \& Fernandes, 1986, M. petronioi Melo, Lobão \& Fernandes, 1986 (TAKINO et al., 1989), M. borellii (Nobili, 1896) (BOND \& BUCKUP, 1982; VerdI, 1996) e $M$. ohione (Smith, 1874) (Truesdale \& Mermilliod, 1979), apresentaram o período reprodutivo durante as épocas em que são registradas as maiores temperaturas da água e do ar.

A temperatura da água atua diretamente sobre a fisiologia dos crustáceos, influenciando no desenvolvimento dos tecidos gonadais (ADIYODI \& ADIYODI, 1970; WEAR, 1974) ou acelerando as diferentes etapas do desenvolvimento pós-embrionário (Boolootian et al., 1959).

TAKINO et al. (1989), ao estudarem as relações entre fatores climáticos e abióticos e o período reprodutivo de $M$. birai e $M$. petronioi, sugeriram que não somente a 
temperatura da água, como também o índice pluviométrico que aumenta a vazão do rio - assim como ocorreu no presente estudo -, e vários outros fatores podem regular o período reprodutivo dessas espécies. TAKINO et al. (1989) verificaram que a maior vazão de um rio proporciona melhores condições para a migração dos camarões, facilitando sua reprodução. ODInetz-Collart \& ENRICONI (1993) observaram que a época de reprodução de Palaemonetes carteri (Gordon, 1935) foi restrita à enchente das águas alagadas anualmente. Portanto, desovar durante as enchentes é uma estratégia reprodutiva muito comum em espécies aquáticas tropicais, favorecendo a dispersão larval longitudinal para jusante, ou lateral nas planícies de inundação (WELCOMME, 1985).

De acordo com THORSON (1950) e SASTRY (1983), fêmeas ovígeras que apresentam pico reprodutivo evidente, como é o caso do presente estudo, onde os picos reprodutivos se concentraram no período chuvoso-quente, geralmente está associado às variações ambientais, como temperatura, a latitude, o fotoperíodo e a disponibilidade de alimentos.

Em relação à maturidade sexual de $M$. jelskii observado no rio São Francisco (Fig. 4), outros autores verificaram a maturidade sexual individual para essa mesma espécie, semelhante ao encontrado no presente estudo. GAMBA (1997) registrou aos 34,5 mm de comprimento total e PAIVA \& BARreto (1960) aos $31,5 \mathrm{~mm}$, enquanto que Mossolin et al. (2013) aos 7,85 mm de comprimento do cefalotórax. José Carlos C. da Silva (dados inéditos) verificou fêmeas maduras aos $6,5 \mathrm{~mm}$, sendo de tamanhos inferiores ao do presente estudo.

Diferenças no tamanho para o início da atividade reprodutiva podem estar relacionadas à oferta de itens alimentares ou estresse ambiental (SASTRY, 1983; CAMPBELL \& EAgles, 1983; Hines, 1989). Segundo NegreirosFrANSOzo et al. (2003), indivíduos que se dispersam em ambientes com condições extremas, podem começar a procriar antecipadamente se os recursos alimentares mostrarem-se escassos ou com qualidade reduzida. Essa antecipação também pode ocorrer devido às diferenças em relação ao teor de sais, que além de induzir a população ao crescimento atrofiado, antecipa a maturidade sexual nos indivíduos de menor porte (DíAz \& CONDE, 1989). As variações no tamanho da maturidade são comuns em crustáceos podendo ser causadas por variações na taxa de crescimento e por vários graus de variabilidade que podem ocorrer sazonalmente ou em diferentes localidades (WENNER et al.,1985). E até mesmo parâmetros ambientais relacionados à latitude podem promover antecipação ou retardo da maturidade nos crustáceos, caracterizando variações entre populações de uma mesma espécie (HINES, 1989).

No caso do presente estudo, devido à menor quantidade de vegetação marginal e por consequência um número reduzido de refúgios, os animais a montante podem ser mais susceptíveis ao estresse, como a predação, e com isso, tendem a atingir a maturidade sexual antecipadamente, quando comparados aos animais a jusante.
Verificou-se que o tamanho das fêmeas interfere no número de ovos transportados. As maiores fêmeas carregam maior quantidade de ovos, devido ao aumento do seu espaço abdominal utilizado para a incubação (ANTUNES \& OSHIRO, 2004). Essa relação direta evidencia um padrão verificado em outros carídeos como $M$ carcinus, M. acanthurus e M. potiuna estudados, respectivamente por LoBÃo et al. (1985), VALENTI et al. (1989) e AnTUnes \& Oshiro (2004).

A fecundidade de $M$. jelskii a montante e a jusante foram muito semelhantes àquelas encontradas para a mesma espécie por GAMBA (1997) (aproximadamente uma média de 35 ovos, variando de 10 a 56, para fêmeas ovígeras com comprimento total entre 34,4 e $45,3 \mathrm{~mm}$ ) e por J. C. da Silva (dados inéditos) (quantidade de ovos: 32,8 \pm 14 ; comprimento do cefalotórax das fêmeas ovígeras nas classes de tamanho de 7 a $12 \mathrm{~mm}$ ); superiores às de Mossolin et al. (2013) (quantidade de ovos: 23,95 $\pm 14,8$; comprimento do cefalotórax das fêmeas ovígeras: 7,85 a $10,70 \mathrm{~mm}$ ) e inferiores ao encontrado por PAIVA \& BARRETO (1960) (aproximadamente uma média de 59 ovos, de 16 a 107, para fêmeas ovígeras com comprimento total de $31,5$ a $44 \mathrm{~mm})$.

De acordo com SASTRY (1983), em crustáceos a energia alocada para a produção de gametas pode vir a proporcionar desde um grande número de pequenos ovos, até uma quantidade reduzida de grandes ovos. Estudos que abordam a fecundidade em palaemonídeos concretizam a hipótese de que $M$. jelskii seja uma das espécies que apresentam um menor número de ovos, com maiores volumes e dimensões (PAIVA \& BARRETO, 1960; GAMBA, 1984; MagalHães, 2000).

O aumento do tamanho dos ovos, com proporcional redução no número de ovos, a redução do número de estágios larvais e da duração de períodos larvais, e um aumento da sobrevivência larval em populações de águas continentais interiores, consiste em um padrão evolutivo que envolve a maioria das espécies de Macrobrachium (JALIHAL et al., 1993). Esse padrão, também pode ser observado em M. potiuna (SouzA \& FontourA, 1996; Müller et al., 1999; LIMA \& OSHIRO, 2000; ANTUNES \& OshIRo, 2004). No entanto, espécies como M. acanthurus (Wiegmann, 1836), M. carcinus (Linnaeus, 1758) e $M$. olfersii (Wiegmann, 1836), apresentam alta fecundidade e menor volume e dimensão dos ovos (VALENTI et al., 1989; Müller et al., 1999; NAZARI et al., 2003).

NAZARI et al. (2003), ao comparar o número e o volume dos ovos de $M$. potiuna e $M$. olfersii, mencionaram que diferenças de fecundidade e volume dos ovos nestas duas espécies de porte semelhantes são indícios de adoção de diferentes estratégias reprodutivas. HAYASHI \& HAMANo (1984), Rabalais \& GoRe (1985) e JALIHAL et al. (1993), ao estudarem características reprodutivas em decápodos, observaram uma associação intrínseca entre os tipos de habitat e as estratégias reprodutivas. Um exemplo desta associação intrínseca é o estudo de HANCOCK (1998), que afirmou que espécies habitantes de ambientes estuarinos produzem grande número de ovos 
pequenos, pois concentrações de sais nesses ambientes reduzem a quantidade de água dos ovos, através de processo osmótico. No entanto, as que habitam ambientes límnicos/ lênticos produzem ovos de números e tamanhos médios. Enquanto espécies que vivem próximas às cabeceiras de riachos, como a espécie em estudo, produzem um reduzido número de volumosos ovos.

De acordo com OH \& HaRTnoll (1999), as condições fisiológicas das fêmeas, estações do ano, latitude e condições ambientais propiciam diferenças em relação ao número de ovos. E segundo Ammar et al. (2001), as variações na estimativa da fecundidade podem ser decorrentes de diferenças no tamanho máximo dos exemplares e das características genéticas próprias das populações de origem. No entanto, fêmeas menores apresentam condições fisiológicas e morfológicas distintas, uma vez que desovam menor número de ovos por apresentarem um reduzido tamanho ovariano e espaço abdominal restrito para a incubação dos mesmos (CLARKE, 1993).

De acordo com alguns autores, o número de ovos carregados por um crustáceo Pleocyemata é relativamente variável para uma mesma classe de tamanho (LoBÃo et al., 1985; NeGReIRos-Fransozo et al., 1992), concordando com os resultados obtidos no presente estudo. PARsOns \& TUCKER (1986) mencionaram que tal fato deve-se à perda acidental dos ovos ou devido à fertilização incompleta, ou ainda, a doenças.

Neste estudo, $M$. jelskii apresentou ovos maiores que aqueles encontrados por PAIVA \& BARReto (1960), Gamba (1997) e J. C. da Silva (dados inéditos).

Nas duas áreas estudadas ocorreu um crescimento maior do maior eixo dos ovos entre as fases I e II do desenvolvimento embrionário (Tab. II). PAIVA \& BARRETO (1960), Gamba (1997), Lima \& Oshiro (2000), Nazari et al. (2003) e MüLLER et al. (2004) também encontraram aumento entre os eixos menor e maior dos ovos no decorrer do período de incubação. De acordo com ANDERSON (1982), o grande aumento do maior eixo dos ovos reflete o crescimento de estruturas embrionárias no eixo cefalocaudal.

O maior tamanho dos ovos carregados pelos camarões a jusante, provavelmente se deve às menores temperaturas da água neste ponto $\left(24,03 \pm 1,11^{\circ} \mathrm{C}\right.$, variando de 22,46 a $\left.25,86^{\circ} \mathrm{C}\right)$ em comparação com a montante $(27,01$ $\pm 1,44^{\circ} \mathrm{C}$, de 24,84 a $\left.29,26^{\circ} \mathrm{C}\right)(\mathrm{t}=5,17 ; \mathrm{p}<0,05)$, já que as águas que saem do reservatório para o Rio são provenientes do fundo da Represa e, portanto mais frias. SAMPAIO \& LóPEZ (2003), também verificaram para essa mesma represa, na região da barragem, a temperatura máxima da água de $31,1^{\circ} \mathrm{C}$ na superfície e a mínima de $21,4^{\circ} \mathrm{C}$ na camada do fundo.

A inferência de que a temperatura da água influencia no tamanho dos ovos de M. jelskii no presente estudo pode ser corroborada por ClARKE et al. (1991) e CLARKE (1992), que em experimentos com carídeos marinhos revelaram que o tamanho dos ovos aumenta com a diminuição das temperaturas, promovendo assim a tendência para que estes animais produzam ovos maiores em altas latitudes.

GIMÉNEZ \& ANGER (2001) verificaram que fêmeas de uma mesma espécie submetidas a condições ambientais idênticas apresentaram variação no tamanho dos ovos, indicando a existência de fatores intrínsecos que regulam estas alterações biométricas. Fatores ambientais como salinidade e temperatura são considerados de maior relevância, para a variabilidade de tamanho dos ovos (Wear, 1974; Steele \& Steele, 1975 e Bas \& Spivak, 2000).

Os dados sobre os aspectos reprodutivos de $M$. jelskii realizado no rio São Francisco, permitiram concluir que as diferenças encontradas nos dois locais de coleta ocorrem devido à quantidade de vegetação marginal e por interferência da barragem, como a temperatura da água liberada no ponto a jusante. A razão sexual desviada para os machos de M. jelskii a jusante ocorre por causa do comportamento das fêmeas de se abrigarem entre as vegetações marginais. A razão sexual desviada para as fêmeas a montante demonstra a proporção real de machos e fêmeas que pode ser considerada para a espécie no rio São Francisco, pois as fêmeas não encontraram, neste ponto, muitos locais para se abrigarem. A diferença no tamanho dos ovos transportados pelas fêmeas a montante e a jusante deve-se, principalmente, à temperatura da água mais baixa proveniente do fundo da represa de Três Marias.

Agradecimentos. Ao Dr. Yoshimi Sato, a CEMIG GT/ CODEVASF (Companhia Energética de Minas Gerais/Companhia de Desenvolvimento do Vale do São Francisco), a UFRRJ (Universidade Federal Rural do Rio de Janeiro) e ao IBAMA/ESEC de Pirapitinga/ MG (Instituto Brasileiro do Meio Ambiente e dos Reursos Naturais Renováveis/Estação Ecológica de Pirapitinga/Minas Gerais).

\section{REFERÊNCIAS BIBLIOGRÁFICAS}

AdIYODI, K. G. \& ADIYODI, R. G. 1970. Endocrine control of reproduction in decapoda crustacea. Biological Review 45:121-165.

AMmar, D.; MüLler, Y. M. R. \& NAZARI, E. M. 2001.Biologia reprodutiva de Macrobrachiumolfersii (Wiegmann, 1836) (Crustacea, Decapoda, Palaemonidae) coletados na Ilha de Santa Catarina, Brasil. Revista Brasileira de Zoologia 18(2):529-537.

Anderson, D. T. 1982. Embryology. In: Abele, L. G. Embryology, Morphology and Genetics: The Biology of Crustacea. New York, Academic Press. v. 2, p.1-44.

Antunes, L. S. \& Oshiro, L. M. Y. 2004. Aspectos reprodutivos do camarão de água doce Macrobrachium potiuna (Müller) (Crustacea, Decapoda, Palaemonidae) na Serra do Piloto, Mangaratiba, Rio de Janeiro, Brasil. Revista Brasileira de Zoologia 21(2):261-266.

Barros-Alves, S. P.; Almeida, A. C.; Fransozo, V.; Alves, D. F. R.; Silva, J. C. \& Сово, V. J. 2012. Population biology of shrimp Macrobrachium jelskii (Miers, 1778) (Decapoda, Palaemonoidea) at the Grande River at northwest of the state of Minas Gerais, Brazil. Acta Limnologica Brasiliensia 24(3):266-275.

BAS, C. C. \& SPIVAK, E. D. 2000. Effect of salinity on embryos of two southwestern atlantic estuarine grapsidae crab species culture in vitro. Journal of Crustacean Biology 20(4):647-656.

Bastos, J. A. M. \& PAIVA, M. P. 1959. Notas sôbre o consumo de oxigênio do camarão "sossego", "Macrobrachium jelskii" (Miers, 1877) Chace \& Holthuis, 1948. Revista Brasileira de Biologia 19(4):413-419.

Beiguelman, B. 2002. Curso prático de bioestatística. 5ed. Ribeirão Preto, Fundação de Pesquisa Científica de Ribeirão Preto. 272p. 
Bond, G. \& Buckup, L. 1982. O ciclo reprodutivo de Macrobrachium borellii (Nobili, 1896) e Macrobrachium potiuna (Müller, 1880) (Crustacea, Decapoda, Palaemonidae) e suas relações com a temperatura. Revista Brasileira de Biologia 42(3):473-483.

Boolootian, R. A. A.; Giese, A. C.; Farmanfarmain, A. \& Tucker, J. 1959. Reproductive cycles of five west coast crabs. Physiological Zoology 32:213-220.

Botelho, E. R. O.; Santos, M. C. F. \& Souza, J. R. B. 2001. Aspectos populacionais do Guaiamum, Cardisoma guanhumi Latreille, 1825, do estuário do Rio Una (Pernambuco - Brasil). Boletim Técnico Científico CEPENE 9(1):123-146.

Campbell, A. \& Eagles, M. D. 1983. Size at maturity and fecundity of rock crabs, Cancer irroratus, from the Bay of Fundy and southwestern Nova Scotia. Fishery Bulletin 81(2):357-362.

Clarke, A. 1992. Reproduction in the cold: Thorson revisited. Invertebrate Reproduction and Development 22:175-184.

1993. Reproductive trade-offs in caridean shrimps. Functional Ecology 7:411-419.

Clarke, A.; Hopkins, C. C. E. \& Nilssen, E. M. 1991. Egg size and reproductive output in the deep-water prawn Pandalus borealis Kroyer, 1938. Functional Ecology 5:724-730.

Collins, P. 2000. A new distribution record for Macrobrachium jelskit (Miers, 1877) in Argentina (Decapoda, Palaemonidae). Crustaceana 73(9):1167-1169.

Díaz, H. \& Conde, J. E. 1989. Population dynamics and life history of the mangrove crab Aratuspisonii (Brachyura, Grapsidae) in a marine environment. Bulletin of Marine Science 45(1):148-163.

Fransozo, A.; Rodrigues, F. D.; Freire, F. A. M. \& Costa, R. C. 2004. Reproductive biology of the freshwater prawn Macrobrachium iheringi (Ortamann, 1897) (Decapoda: Caridea: Palaemonidae) in the Botucatu region, São Paulo, Brazil. Nauplius 12(2):119-126.

Furota, T. 1996. Life cycle studies on the introduced spider crab Pyromaia tuberculata (Lockington) (Brachyura:Majidae). Journal of Crustacean Biology 16:71-76.

GAmBA, A. L. 1984. Different egg - Associated and larval development characteristics of Macrobrachium jelskii and Macrobrachiumam azonicum (Arthropoda: Crustacea) in a Venezuelan continental lagoon. International Journal of Invertebrate Reproduction Development 7:135-142.

1997. Biologia reproductive de Macrobrachium jelskii (Miers, 1877) y Macrobrachium amazonicum (Heller, 1862) en Vanezuela (Crustacea, Decapoda, Palaemonidae). Acta Cientifica Venezolana 48:19-26.

GimÉnEZ, L. \& ANGER K. 2001. Relationships among salinity, egg size, embryonic development, and larval biomass in the estuarine crab Chasmagnathus granulata Dana, (1851). Journal Experimental of Marine Biology and Ecology 260:241-257.

HANCOCK, M. A. 1998. The relationship between egg size and embryonic and larval development in the freshwater shrimp Paratya australiensis (Kemp) (Decapoda, Atyidae). Freshwater Biology 39:715-723.

Hayashi, K. I. \& Hamano, T. 1984. The complete larval development of Caridina japonica De Man (Decapoda, Caridea, Atyidae) reared in the laboratory. Zoological Science 1:571-589.

Hines, A. H. 1989. Geographic variation in size at matury in brachyuran crabs. Bulletin of Marine Science 45(2):356-368.

Howard, R. K. \& Lowe, K. W. 1984. Predation by birds as a factor influencing the demography of an intertidal shrimp. Journal of Experimental Marine Biology and Ecology 74:53-66.

Jalihal, D. R.; SANKolli, K. N. \& ShenOY, S. 1993. Evolution of larval development patterns and the process of freshwaterization in the prawn genus Macrobrachium Bate, 1868 (Decapoda, Palaemonidae). Crustaceana 65:365-376.

Lima, E. A. C. \& Oshiro, L. M. Y. 2000. Distribuição, abundância e biologia reprodutiva de Macrobrachium potiuna (Müller, 1880) (Crustacea, Decapoda, Palaemonidae) do Rio Paraíba do Sul, RJ. Rio de Janeiro. Acta Biologica Leopoldensia 22(1):67-77.

Lobão, V. L.; Valenti, W. C. \& Mello J. T. C. 1985. Fecundidade em Macrobrachium carcinus, do Rio Ribeira do Iguape. Boletim do Instituto de Pesca 12:1-8.

MagalHÃES, C. 2000. Abbreviated development of Macrobrachium jelskii (Miers, 1877) (Crustacea: Decapoda: Palaemonidae) from the
Rio Solimões foodplain, Brazil, reares in the laboratory. Nauplius 8(1): 1-14.

Mantelatto, F. L. M. \& Barbosa, L. R. 2005. Populations structure and relative growth of freshwater prawn Macrobrachium brasiliense (Decapoda, Palaemonidae) from São Paulo State, Brazil. Acta Limnologica Brasiliensia 17(3):245-255.

Melo, G. A. S. 2003. Manual de Identificação dos Crustacea Decapoda de água doce do Brasil. São Paulo, Loyola, Centro Universitário São Camilo, Museu de Zoologia, Universidade de São Paulo. 430p.

Montoya, J. V. 2003. Freshwater shrimps of the genus Macrobrachium associted with roots of Eichhornia crassipes (water Hyacinth) in the Orinoco Delta (Venezuela). Carribean Journal of Science 39(1):155-159.

Mossolin, E. C.; Peiró, D. F.; Rossingnoli, M. O.; Rajab, L. P. \& Mantelatto, F. L. 2013. Population and reproductive features of the freshwater shrimp Macrobrachium jelskii (Miers, 1877) from São Paulo state, Brazil. Acta Scientiarum, Biological Sciences 35(3):429-436.

Müller, Y. M. R.; NaZari, E. M.; Ammar, D.; Ferreira, E. C.; Beltrame, I. T. \& PACHeCO, C. 1999. Biologia dos Palaemonidae (Crustacea, Decapoda) da bacia hidrográfica de Ratones, Florianópolis, Santa Catarina, Brasil. Revista Brasileira de Zoologia 16(3):629-636.

Müller, Y. M. R.; Ammar, D. \& Nazari, E. M. 2004. Embryonic development of four species of palaemonid prawns (Crustacea, Decapoda): pre-naupliar, naupliar and post-naupliar periods. Revista Brasileira de Zoologia 21(1):27-32.

Nazari, E. M.; Simões-Costa, M. S.; Müller, Y. M. R.; Ammar, D. \& Dias, M. 2003. Comparisons of fecundity, egg size, and egg mass volume of the freshwater prawns Macrobrachium potiuna e Macrobrachium olfersi (Decapoda, Palaemonidae). Journal of Crustacean Biology 23(4):862-868.

Negreiros-Fransozo, M. L.; Colpo, K. D. \& Costa, T. M. 2003. Allometric growth in the fiddler crab Uca thayeri (Brachyura, Ocypodidae) from a subtropical mangrove. Crustaceana 23(2):273279.

Negreiros-Fransozo, M. L.; Fransozo, A.; Mantelatto, F. L. M.; Nakagaki, J. M. \& Spilborghs, M. C. F. 1992. Fecundity of Paguristes tortugae (Schimitt, 1933) (Crustacea, Decapoda, Anomura) in Ubatuba, São Paulo, Brazil. Revista Brasileira de Biologia 52:547-553.

Odinetz-Collart, O. \& Enriconi, A. 1993. Estratégia reprodutiva e alguns aspectos demográficos do camarão Palaemonete scarteri (Gordon, 1935) na Amazônia Central, Rio Negro. Acta Amazonica 23(2-3):227-243.

OH, C. W. \& Hartnoll, R. G. 1999. Size at sexual maturity, reproductive output, and seasonal reproduction of Philocheras trispinosus (Decapoda) in Port Erin Bay, Isle of Man. Journal of Crustacean Biology 19(2):252-259.

PAiva, M. P. \& BARreto, V. A. 1960. Notas sobre a biologia do camarão "sossego" Macrobrachium jelskii (Miers, 1877) numa pequena bacia potamográfica do nordeste brasileiro. Revista Brasileira de Biologia 20(2):121-129.

Parsons, D. G. \& Tucker, G. E. 1986. Fecundity of northern shrimp Pandalus borealis (Crustacea, Decapoda), in areas of northwest Atlantic. Fishery Bulletin 84:549-558.

Pinheiro, M. A. A. \& Fransozo, A. 2002. Reproductive dynamics of the speckled swimming crab Arenaeus cribrarius (Lamarck, 1818) (Brachyura, Portunidae), on the north coast of São Paulo State, Brazil. Journal of Crustacean Biology 22(2):416-428.

Rabalais, N. N. \& Gore, R. H. 1985.Abbreviated development in decapods. In: SCHRAM, F. R. ed. Crustacean issues: Larval growth. 2ed. Rotterdam, A. A. Balkema, p.67-126.

SAmpaio, E. V. \& López, C. M. 2003. Limnologia física, química e biológica da represa de Três Marias e do São Francisco. In: GodINHO, H. P. \& Godinho, A. L. orgs. Águas, peixes e pescadores do São Francisco das Minas Gerais. Belo Horizonte, PUC Minas, p.71-92.

SASTRY, A. N. 1983. Ecological aspects of reproduction. In: VERnBERG, F. J. \& VernberG, W. B. The Biology of Crustacea: Enviromental Adaptations. 8ed. New York, Academic Press, p.179-255.

SouzA, G. D.; Barros, M. P. \& Braun, A. S. 1996. Densidade populacional de Macrobrachium potiuna (Müller, 1880) (Decapoda, Palaemonidae) 
no Arroio Sapucaia, Localidade Morro Agudo, Município de Gravataí, RS. Nauplius 4:61-72.

Souza, G. D. \& Fontoura, N. F. 1996. Reprodução, longevidade e razão sexual de Macrobrachium potiuna (Muller, 1880) (Crustacea, Decapoda, Palaemonidae) no Arroio Sapucaia, Município de Gravataí, Rio Grande do Sul. Nauplius 4:49-60.

Steele, D. H. \& Steele, V. J. 1975. Egg size and duration of embryonic development in Crustacea. Internationale Revue der Gesamten Hydrobiologie 60(5):711-715.

TAKIno, M.; LobÃo, V. L.; GolubeFF, T. \& Lombardi, J. V. 1989. Relações entre fatores climáticos e abióticos e o período reprodutivo das populações de Macrobrachium birai Lobão, Melo \& Fernandes e de Macrobrachium petronioi Melo, Lobão \& Fernandes (Decapoda, Palaemonidae) do Rio Branco, Cananéia, São Paulo, Brasil. Boletim do Instituto de Pesca 16(1):67-80.

Thorson, G. 1950. Reproductive and larval ecology of marine bottom invertebrates: Biology Revis. Cambridge Philosophy Society 25:1-45.

Truesdale, F. M. \& Mermilliod, W. J. 1979. The river shrimp Macrobrachium ohione (Smith) (Decapoda, Palaemonidae): its abundance, reproduction and growth in the Atchafalaya river basin of Louisiana, U.S.A. Crustaceana 36(1):61-73.

Valenti, W. C.; Mello, J. T. C. \& Lobão, V. L. 1989. Fecundidade em Macrobrachium acanthurus do Rio Ribeira do Iguape (Crustacea, Decapoda, Palaemonidae). Revista Brasileira de Zoologia 6(1):9-15.
VAzzoler, A. E. A. M. 1981. Manual de métodos para estudos biológicos para populações de peixes, reprodução e crescimento. Brasília, CNPq. 108p.

Verdi, A. C. 1996. Ciclo anual de reproducción del camarón dulceacuícola Macrobrachium borellii (Nobili, 1896) (Crustacea, Caridea, Palaemonidae). Revista Brasileira de Biologia 56(3):561-568.

Wear, R. G. 1974. Incubation in British decapod Crustacea, and the effects of the temperature on the rate and success of embryonic development. Journal of the Marine Biological Association of the United Kingston 54:745-762.

Welcomme, R. L. 1985. River Fisheries. Roma, F.A.O. Fisheries Technical Paper. V. 262. 330p.

WENNER, A. M. 1972. Sex ratio as a function of size in marine crustacean. The American Naturalist 106:321-351.

Wenner, A. M.; Page, H. M. \& Siegel, P. R. 1985. Variation in size at onset of egg production. In: WenNer, A. M. Factors in adult growth. Rotterdam, A. A. Balkema, p.149-163.

Wilson, M. F. \& Pianka, E. R. 1963. Sexual selection, sex ratio and mating system. American Nature 97:405-407.

ZAR, J. H. 2010. Biostatistical analysis. New Jersey, Prentice-Hall. 994p. 